\title{
Participation of the female pronucleus derived from the second polar body in full embryonic development of mice
}

\author{
T. Wakayama ${ }^{\text {* }}$, Y. Hayashi ${ }^{1}$ and A. Ogura ${ }^{2}$ \\ ${ }^{1}$ Department of Veterinary Anatomy, Faculty of Agriculture, The University of Tokyo, Bunkyo-ku, \\ Tokyo 113, Japan; and ${ }^{2}$ Department of Veterinary Science, Japan's National Institute of Health, \\ Shinjuku-ku, Tokyo, Japan
}

\begin{abstract}
The second polar body (2PB), extruded from metaphase II oocytes after fertilization or oocyte activation, has a haploid set of female chromosomes like its sister, the fertilized (activated) oocyte. In the present study, the female pronucleus of fertilized mouse oocytes (zygotes) was replaced with the $2 \mathrm{~PB}$ nucleus from the same or different oocytes to examine the developmental potential of the $2 \mathrm{~PB}$ nucleus. When the female pronucleus (FPN) was synchronously (FPN and $2 \mathrm{~PB}$ were same age) replaced with the $2 \mathrm{~PB}$ nucleus, the rate of reconstructed zygotes developing to blastocysts decreased with the age of donors and recipients (from $70 \%$ at $20-21 \mathrm{~h}$ to $15 \%$ at $26-27 \mathrm{~h}$ after hCG injection). When nuclei were replaced asynchronously (FPN and 2PB were of different ages), a higher developmental rate to blastocysts was obtained with young recipient zygotes ( $20 \mathrm{~h}$ after hCG injection) than with aged recipient zygotes ( $24 \mathrm{~h}$ after $\mathrm{hCG}$ injection) $(64 \%$ versus $20 \%, P<0.01$ ) irrespective of the age of the $2 \mathrm{~PB}$. In this second group of embryos, in which nuclei were replaced asynchronously, the $2 \mathrm{~PB}$ nuclei were prematurely condensed at the time of first mitosis. These findings indicate that after being extruded from the oocytes the cell cycle of the $2 \mathrm{~PB}$ progressed more slowly than did that of the zygote. After the transfer of reconstructed embryos into pseudopregnant females, normal pups with an expected coat colour were born, indicating the competence of the $2 \mathrm{~PB}$ chromosomes for full embryo development.
\end{abstract}

\section{Introduction}

The meiotic division of mammalian oocytes differs in several significant ways from that of male germ cells. As a result of male meiotic divisions of single diploid cells (spermatogonia), sets of four round spermatids with an equal volume of cytoplasm are formed. However, the female germ cells (oocytes) undergoing meiotic division sequentially extrude two small polar bodies from the large main egg body. These polar bodies never participate in embryo development and degenerate some time during the preimplantation period (Gilbert, 1991; Evsikov and Evsikov, 1995). The second polar body $(2 \mathrm{~PB})$, which is extruded after fertilization or parthenogenetic activation, has a haploid set of female chromosomes like its sister, the fertilized (or activated) oocyte. Therefore, the 2PBs have been extensively used to assess the numerical or structural chromosomal abnormalities of the developing embryo in clinical and basic research (Dyban et al., 1992; Wheeler et al., 1995).

So far, many attempts have been made to examine the ability of the $2 \mathrm{~PB}$ to support embryo development. Zygotes

\footnotetext{
*Correspondence should be sent to: Department of Anatomy and Reproductive Biology, School of Medicine, University of Hawaii, 1951 East-West Road, Honolulu, Hawaii 96822 USA.

Received 24 October 1996.
}

fused with the $2 \mathrm{~PB}$, or diploid parthenogenetic embryos produced by inhibition of the extrusion of the $2 \mathrm{~PB}$, readily developed to the blastocyst stage in vitro (Opas, 1977; Modlinski and McLaren, 1980; Borsuk, 1982). However, the conclusive evidence for the hypothesis that the chromosomes of the $2 \mathrm{~PB}$ have the same developmental potential as their sister chromatids is to show full-term development of embryos that contain the $2 \mathrm{~PB}$ chromosomes. In the present study, diploid mouse zygotes were reconstructed by replacing the female pronuclei with the nucleus of the $2 \mathrm{~PB}$ to examine their developmental ability in vitro and in vivo.

\section{Materials and Methods}

\section{Preparation of donor $2 P B$ s and recipient embryos}

Recipient pronuclear embryos were obtained from B6CBAFI $(\mathrm{C} 57 \mathrm{BL} / 6 \times \mathrm{CBA})$ females mated with $\mathrm{DBA} / 2$ males. Donor $2 \mathrm{PBs}$ were obtained from the same recipient embryos, or $\mathrm{DBA} / 2$ females mated with $\mathrm{DBA} / 2$ males. All females were injected with 5 iu pregnant mares' serum gonadotrophin (PMSG) and 5 iu hCG at a $48 \mathrm{~h}$ interval, and caged with males overnight. The pronuclear embryos were collected from the oviducts $15 \mathrm{~h}$ after hCG injection. They were freed of cumulus cells by treatment with hyaluronidase $(0.1 \%, w / v)$ in $\mathrm{mKRB}$ 

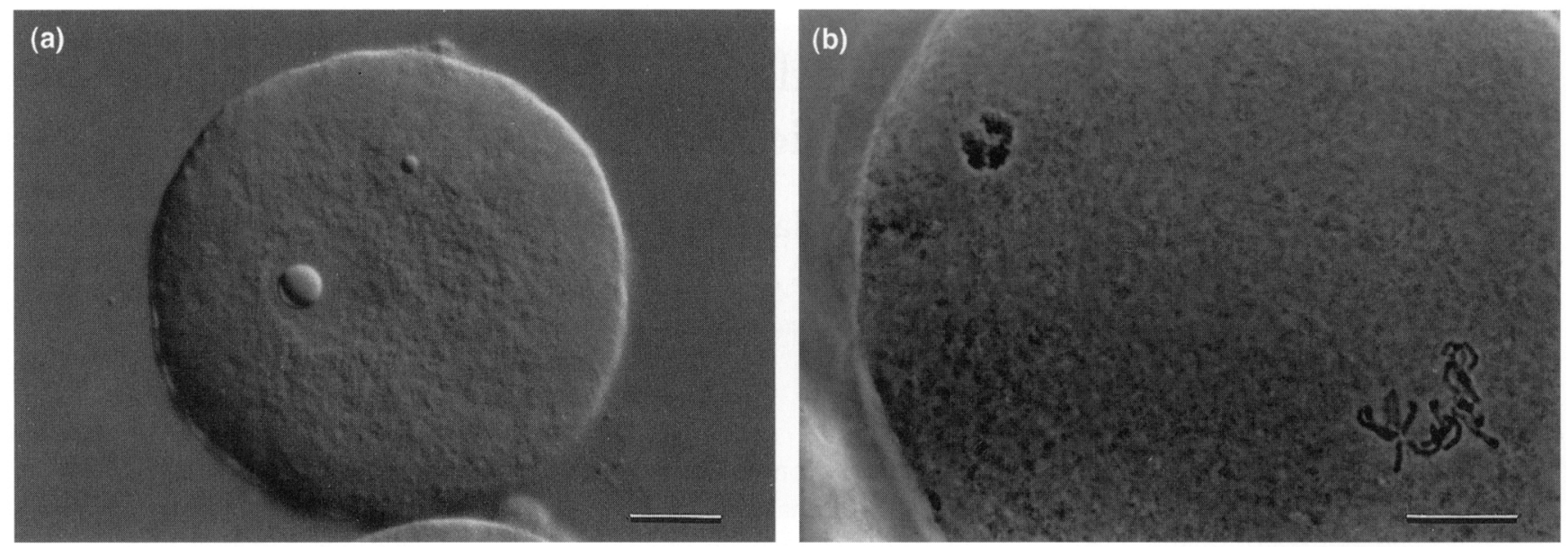

Fig. 1. (a) Reconstructed mouse embryo that was stained with acetoorcein. These embryos had a large male pronucleus and a small female pronucleus derived from a second polar body. Scale bar represents $25 \mu \mathrm{m}$. (b) Reconstructed mouse embryo formed from fusion between an aged zygote and an aged second polar body. The prematurely condensed chromosomes can be observed next to the fully condensed paternal chromosome mass. Scale bar represents $15 \mu \mathrm{m}$.

medium (Toyoda et al., 1974) and washed three times with fresh mKRB medium.

\section{Nuclear transfer and examination of the 2PB-derived pronuclei}

All manipulations were performed under an inverted microscope with Nomarski's contrast optics and micromanipulators. The $2 \mathrm{PBs}$ and female pronuclei were removed from appropriate zygotes through the zonae pellucidae, which had been partially dissected with a fine glass needle (Tsunoda et al, 1986). The female pronucleus was chosen on the basis of its proximal location to the $2 \mathrm{~PB}$ and its small size relative to the other pronucleus (male pronucleus). Nuclear transfer was undertaken in a small drop of mKRB containing cytochalasin $\mathrm{B}(5 \mu \mathrm{g}$ $\mathrm{ml}^{-1}$ ). The 2PBs were inserted into the perivitelline space of the recipients and the $2 \mathrm{~PB}$-zygote pairs were placed in a drop $(10 \mu \mathrm{l})$ of the fusion medium $\left(300 \mathrm{mmol}\right.$ mannitol $\mathrm{I}^{-1}$, $0.05 \mathrm{mmol} \mathrm{CaCl}_{2} \mathrm{1}^{-1}, 0.1 \mathrm{mmol} \mathrm{MgSO}_{4} \mathrm{I}^{-1}, 5 \mathrm{mg}$ polyvinylpyrrolidone $\mathrm{ml}^{-1}$ ) between the electrodes of a circular electrofusion chamber (Shimadzu, Kyoto). The width and depth of the electrode gap were 0.5 and $2.0 \mathrm{~mm}$, respectively. Electrofusion of the $2 \mathrm{~PB}$ and nucleated zygotes was induced by applying $20 \mathrm{~V} \mathrm{~cm}^{-1} \mathrm{AC}$ for $30 \mathrm{~s}, 3000 \mathrm{~V} \mathrm{~cm}^{-1} \mathrm{DC}$ for $10 \mu \mathrm{s}$, and $20 \mathrm{~V} \mathrm{~cm}^{-1} \mathrm{AC}$ for $90 \mathrm{~s}$, consecutively.

At 3-13 h after electrofusion, the reconstructed embryos were washed, whole-mounted on glass slides, fixed with $2.5 \%$ $(\mathrm{v} / \mathrm{v})$ glutaraldehyde for $10 \mathrm{~min}$, and then stained with $0.25 \%$ (w/v) acetoorcein (Yanagida et al., 1991). The embryos were examined under a phase-contrast microscope.

\section{Assessment of embryo development in vitro}

At 30 min after application of fusion pulse, embryos were examined under a dissecting microscope and those fused were washed and cultured further for $96 \mathrm{~h}$. Two series of experiments were carried out to assess the developmental ability of reconstructed embryos. In the first experiment, the $2 \mathrm{~PB}$ nuclei were transferred into synchronous recipients (the female pro- nucleus and the second polar body were the same age) between $20 \mathrm{~h}$ and $27 \mathrm{~h}$ after hCG injection. In the second experiment, synchronous and asynchronous (the female pronucleus and the second polar body were of different ages) nuclear transfer was performed using $2 \mathrm{PBs}$ and recipient zygotes at either $20 \mathrm{~h}$ (young) or $24 \mathrm{~h}$ (aged) after hCG $(2 \times 2$ factorial design).

\section{Assessment of embryo development in vivo}

After $96 \mathrm{~h}$ of culture, compacted morulae or blastocysts were transferred to the day 3 uteri of pseudopregnant ICR females. In this experiment, donor embryos were from DBA (aabbCCdd) females $\times$ DBA males and recipient embryos were from B6CBAF1 (AaBBCCDD) females $\times$ DBA males. Therefore, original recipient embryos would develop to offspring with black (aa) or agouti (heterozygous) coat colour and reconstructed embryos would develop to offspring with the DBA coat colour (dd, diluted). Nineteen days after transfer, recipient females were killed and the uteri were examined for live or dead fetuses. Live fetuses were raised by other lactating foster mothers (ICR mice).

\section{Statistical analysis}

The data were analysed by Fisher's exact probability test.

\section{Results}

\section{Examination of the 2PB-derived pronuclei}

All reconstructed embryos examined (both synchronous and asynchronous) had a well developed pronucleus (male pronucleus) and a small pronucleus (2PB-derived female pronucleus) (Fig. Ia). The 2PB-derived female pronuclei remained small until the first mitotic division (about $34 \mathrm{~h}$ after hCG injection). 
Table 1. Development in vitro of mouse embryos after synchronous transfer of the second polar body at various times after hCG injection (embryos studied 4 days after injection)

\begin{tabular}{lcrll}
\hline & & \multicolumn{3}{c}{ Number (\%) of embryos } \\
\cline { 4 - 5 } $\begin{array}{l}\text { Time after hCG } \\
\text { injection }(\mathrm{h})\end{array}$ & $\begin{array}{l}\text { Number } \\
\text { of eggs }\end{array}$ & Two-cell & Eight-cell & Blastocyst \\
\hline $20-21$ & 27 & $7(25.9)$ & $1(3.7)$ & $19(70.4)$ \\
$22-23$ & 34 & $19(55.9)$ & $0(0)$ & $15(44.1)$ \\
$24-25$ & 33 & $23(69.7)$ & $0(0)$ & $10(30.3)^{*}$ \\
$26-27$ & 27 & $22(81.5)$ & $1(3.7)$ & $4(14.8)^{* *}$ \\
& & & & \\
\hline
\end{tabular}

${ }^{*} P<0.05,{ }^{* *} P<0.01$ (compared with values at $20-21 \mathrm{~h}$ ).

Table 2. Development in vitro of mouse embryos after synchronous and asynchronous transfer of the second polar body (2PB) after hCG injection (embryos studied 4 days after injection)

\begin{tabular}{|c|c|c|c|c|c|}
\hline \multirow{2}{*}{$\begin{array}{l}\text { Age of male } \\
\text { pronucleus } \\
\text { (h) }\end{array}$} & \multirow{2}{*}{$\begin{array}{l}\text { Age of } \\
2 \mathrm{~PB}(\mathrm{~h})\end{array}$} & \multirow{2}{*}{$\begin{array}{l}\text { Number } \\
\text { of eggs }\end{array}$} & \multicolumn{3}{|c|}{ Number $(\%)$ of embryos } \\
\hline & & & Two-cell & Eight-cell & Blastocys \\
\hline 20 & 20 & 32 & $11(34.4)$ & $2(6.3)$ & $19(60)$ \\
\hline 20 & 24 & 23 & $6(26.1)$ & $1(4.3)$ & $16(65)$ \\
\hline 24 & 20 & 42 & $30(71.4)$ & $2(4.8)$ & $10(20)^{*}$ \\
\hline 24 & 24 & 84 & $69(82.1)$ & $0(0)$ & $15(18)^{*}$ \\
\hline
\end{tabular}

${ }^{*} P<0.05$ (compared with value when male pronucleus was $20 \mathrm{~h}$ old).

\section{Embryo development in vitro after synchronous nuclear transfer}

Most embryos reconstructed by synchronous nuclear transfer could develop to the two-cell stage, irrespective of the time of nuclear transfer (20-27 h after hCG injection) (Table 1). However, later embryonic development was significantly affected by the time of nuclear transfer. The best result was obtained when nuclei were transferred $20-21 \mathrm{~h}$ after hCG injection. The developmental rate gradually decreased with age of donors and recipients.

\section{Embryo development in vitro after asynchronous nuclear transfer}

For determination of the cause of poor embryo development after synchronous nuclear transfer of aged $2 \mathrm{~PB}$ nuclei and aged recipient zygotes, asynchronous nuclear transfer was undertaken with young ( $20 \mathrm{~h}$ after hCG injection) and aged ( $24 \mathrm{~h}$ after hCG injection) donors and recipients. Higher developmental rates to the eight-cell and blastocyst stages were obtained with young recipients than with aged recipients, irrespective of the age of the donor 2PBs (Table 2). Reconstructed embryos were cytologically examined at metaphase of the first mitotic division. When young zygotes were fused with young $2 \mathrm{PBs}$, a normal metaphase plate with maternal and paternal chromosomes of mature configuration was formed. In contrast, when aged zygotes were fused with aged $2 \mathrm{PBs}$, the $2 \mathrm{~PB}$ chromosomes that were prematurely condensed were observed in the vicinity of a fully condensed paternal chromosome mass (Fig. 1b).

\section{Development of reconstructed embryos in vivo}

In total, 30 synchronously reconstructed embryos that developed to morulae or blastocysts were transferred to six pseudopregnant females. All six females became pregnant and 18 pups were obtained. One pup was already dead at the time of Caesarian section and three pups failed to be raised by foster mothers. The remaining 14 pups were weaned and grew normally. They all had the DBA/2 coat colour and were shown to be fertile.

\section{Discussion}

In mammals, meiosis in females always starts early in life, often before birth, and is arrested at the first meiotic division. After puberty, a very small number of these oocytes are selected to prepare for fertilization and, as a result, the remaining abundant oocytes are wasted (Yanagimachi, 1994). Superovulation by hormone treatment and maturation techniques for oocytes in vitro have been developed for many mammals to rescue these oocytes that normally degenerate. Another characteristic of female gamete production is its uneven meiotic division. Here again, as a result of the extrusion of polar bodies, three out of four chromatids are wasted. The present study first demonstrated that the nucleus of the $2 \mathrm{~PB}$, which never participates in normal embryo development, potentially has the ability to support full embryo development. We used the laboratory mouse as a model, but it is very probable that the same technique can be applied to other species because in mammals the $2 \mathrm{PBs}$ usually persist for more than $24 \mathrm{~h}$ after fertilization (Evsikov and Evsikov, 1995).

In this study, we exchanged the nuclei of $2 \mathrm{~PB}$ and zygotes at different ages after hCG injection. In the first experiment, synchronous nuclear transfer was performed between zygotes and $2 \mathrm{PBs}$ obtained $2 \mathrm{I}-27 \mathrm{~h}$ after hCG injection. The ability of reconstructed zygotes to develop to blastocysts apparently decreased with time after hCG injection. Two possible reasons can be considered for this dependence on age. First, the developmental rates of zygotes and $2 \mathrm{PBs}$ did not progress in parallel, that of the $2 \mathrm{~PB}$ was probably slower. This might lead to asynchronous development of the male pronucleus and the 2PB-derived female pronucleus within a zygote after nuclear transfer. Second, it is probable that the nucleus of the $2 \mathrm{~PB}$ gradually loses its developmental ability after being extruded from the oocyte. These possibilities were examined in the second experiment by performing synchronous and asynchronous nuclear transfer using $2 \mathrm{PBs}$ and recipient zygotes at either $20 \mathrm{~h}$ (young) or $24 \mathrm{~h}$ (aged) after hCG injection $(2 \times 2$ factorial design). The best result was obtained with young recipient zygotes, irrespective of the age of donor 2PBs. This finding clearly demonstrates that the nuclei of the $2 \mathrm{PBs}$ did not lose their developmental ability, at least within the age range tested, and that the cell cycle of the $2 \mathrm{PBs}$ progressed more slowly than that of normal zygotes. In aged recipient zygotes, the 2PBderived female pronuclei did not seem to catch up developmentally with the male pronuclei, because the female pronuclei condensed prematurely at the first mitosis. The reconstructed embryos probably entered the first mitosis according to the recipients' schedule; in early embryonic cells, there is no 
feedback machinery for detecting the completion of DNA replication (Murray and Hunt, 1993). It is thought that cell division before completion of DNA replication inevitably leads to chromosome damage. This may explain the poor embryonic development after the transfer of $2 \mathrm{PBs}$ into aged recipient zygotes. Premature condensation of the $2 \mathrm{~PB}$ nucleus was also reported by Evsikov and Evsikov (1994) who fused the 2PBs with intact (non-enucleated) zygotes $21-24 \mathrm{~h}$ after hCG injection. In their study, development of $2 \mathrm{~PB}$-containing embryos to blastocysts was also low (51\%) compared with that of zygotes that received another female pronucleus $(100 \%)$. Their finding and ours clearly indicate the importance of synchronizing the female and male pronuclei for subsequent embryo development.

The fact that the mouse $2 \mathrm{~PB}$ supported full-term embryo development indicates its potential as an alternative source of female chromosomes. As the $2 \mathrm{PBs}$ are smaller celis than oocytes, they can be cryopreserved more easily. A limited gene pool is a general characteristic of most endangered species. In future, in vitro fertilization technology for species conservation may be combined with a frozen $2 \mathrm{~PB}$ resource with genetic diversity. In human infertility clinics, the use of the $2 \mathrm{~PB}$ might avoid the motherhood problems caused by oocyte donation, although ooplasm donation would raise another question about possible mechanical damages to the oocytes. In every attempt in animal species and humans to transfer the 2PB nuclei, their synchronization with the male pronuclei would be crucial to clinical success, as demonstrated in this study.

This study was supported by grants from the fellowship of the Japan Society for the Promotion of Science (T. Wakayama), the Japanese Ministry of Education, Science, Sports and Culture (A. Ogura) and the Japanese Ministry of Health and Welfare (A. Ogura).

\section{References}

Borsuk E (1982) Preimplantation development of gynogenetic diploid mouse embryos Journal of Embryology and Experimental Morphology 69 215-222

Dyban AP, Dutter PD, Dozortsev D and Verlinsky Y (1992) Visualization of second polar body chromosomes in fertilized and artificial activated mouse oocytes treated with okadaic acid Journal of Assisted Reproduction and Genetics 95 572-579

Evsikov AV and Evsikov SV (1994) Preimplantation development of manipulated mouse zygotes fused with the second polar bodies: a cytogenetic study International Journal of Developmental Biology 38 725-730

Evsikov AV and Evsikov SV (1995) A study of the first and second polar bodies in mouse oogenesis Russian Journal of Developmental Biology 26 196-200

Gilbert S (1991) The saga of the germ line. In Developmental Biology (3rd Edn) pp 788-825 Sinauer Sunderland, MA

Modlinski J and McLaren A (1980) A method for visualizing the chromosomes of the second polar body of the mouse egg Journal of Embryology and Experimental Morphology 60 93-97

Murray A and Hunt T (1993) Checkpoints and feedback controls. In The Cell Cycle pp 135-152 Oxford University Press, Oxford

Opas J (1977) Effects of extremely low osmolarity on fertilized mouse eggs Journal of Embryology and Experimental Morphology 37 65-77

Toyoda $Y$ and Chang MC (1974) Fertilization of rat eggs in vitro by epididymal spermatozoa and the development of eggs following transfer Journal of Reproduction and Fertility 36 9-22

Tsunoda Y, Yasui T, Nakamura K, Uchida T and Sugie T (1986) Effect of cutting zonae pellucidae on the pronuclear transplantation in the mouse Journal of Experimental Zoology 240 119-125

Wheeler MB, Noble JA and Jarrell VL (1995) Production of live offspring with predicted genotypes using PCR-RFLP analysis of polar bodies from mouse oocytes Molecular Reproduction and Development 40 267-272

Yanagida K, Yanagimachi R, Perreault SD and Kleinfeld RG (1991) Thermostability of sperm nuclei assessed by microinjection into hamster oocytes Biology of Reproduction 44 440-447

Yanagimachi R (1994) Mammalian fertilization. In The Physiology of Reproduction (2nd Edn) pp 189-317 Eds E Knobil and JD Neill. Raven Press, New York 